\title{
Aortic stenosis evaluation: Novel tools to define the complexity
}

\author{
Raveen S. Pal
}

Kingston General Hospital, Kingston, ON, Canada

\section{Article p. 613}

Aortic valve stenosis (AS) is the most common valvular disease pathology in developed countries affecting over $5 \%$ of the population over the age of 75 [1]. Age is also the most significant risk factor in the development of aortic stenosis. As the global population ages [2], AS rates will continue to increase around the world. Some of the challenges that have surfaced in AS management are appropriate diagnosis of stenosis severity and timing of intervention. Patients who develop severe AS are monitored closely for the development of symptoms or the development of left ventricle (LV) function decline. International guidelines $[3,4]$ currently recommend intervention for severe AS once symptoms develop or when there is a decline in $\mathrm{LV}$ ejection fraction (LVEF). As the LV faces an increasing load from the fixed valve obstruction, it is critical that a decline in LV systolic function is not missed. Once missed, the risk of intervention for severe AS rises sharply.

In the present study, Hita et al. [5] demonstrate the use of LV diastolic function and increase in LV end-diastolic pressure (LVEDP) as markers of LV systolic function decline in patients with symptomatic AS. Twenty-six symptomatic patients with severe AS were evaluated prior to cardiovascular surgery with echocardiography and invasive catheterization. Patients with normal LVEDP (less than $15 \mathrm{~mm} \mathrm{Hg}$ ) were compared to patients with elevated LVEDP (over $15 \mathrm{~mm} \mathrm{Hg}$ ). Patients with elevated LVEDP were further subdivided into without coronary artery disease (CAD) or with CAD. Their data show that patients with increased LVEDP also had LV stiffness, measured as a ratio of LV pressure to LV end diastolic volume, and lower systolic longitudinal strain. They also showed that these hemodynamic changes were associated with histologic changes including a higher myocyte cross sectional and collagen volume. These findings suggest that severe AS with compensated LV function, defined by normal LVEDP, is hemodynamically and structurally different from decompensated severe AS.

This work has limitations including the small patient sample size. The study had 26 patients with 7 patients in two groups and 12 in the third group. Further, there were significant differences in the aortic valve gradient between the groups, which may independently account for the differences observed in LVEDP and LV stiffness or diastolic function.

This paper supports the concept that using LVEF to monitor LV systolic function may be too blunt a tool to guide the timing of intervention for severe AS. This study's findings are in keeping with the work of other researchers who have proposed the use of 2-dimensional (2D) longitudinal LV strain $[6,7]$ as a marker of future systolic dysfunction, in patients with severe AS, when LVEF is still preserved. Early identification of declining LV function is an important concern that is necessary in many areas of current medical care. These findings may be generalized to the monitoring of $\mathrm{LV}$ function in a wide range of conditions such as other valvular heart disease, like mitral regurgitation, side effects of chemotherapeutic agents, and degenerative cardiomyopathies.

This work raises the interesting question of whether we need to shift our focus to earlier diagnosis of impending LV dysfunction, instead of waiting for LV dysfunction to occur, prior to inter-

Address for correspondence: Dr Raveen S. Pal, Kingston General Hospital, Kingston, ON, 76 Stuaft St. AW3, K7L 4N6, Canada, tel/fax: 613-549-6666, x 6245, e-mail: rs.pal@queensu.ca

Received: 29.08.2015

Accepted: 09.11.2015 
ventions. The most appropriate tools to diagnose impending LV dysfunction are still to be defined. The use of LVEDP, LV diastolic function, and 2D strain have all been explored in this setting. Further work is being done on the added value of $3 \mathrm{D}$ echocardiography for LV volume and function assessments. With this paper, authors Hita et al. [5] have added an interesting layer to this discussion.

\section{Conflict of interest: None declared}

\section{References}

1. Baumgartner H, Hung J, Bermejo J et al. Echocardiographic assessment of valve stenosis: EAE/ASE recommendations for clinical practice. J Am Soc Echocardiogr, 2009; 22: 1-23.

2. WHO update. http://www.who.int/ageing/en/. Visited July 13, 2015.
3. Nishimura R, Otto C, Bonow R et al. 2014 AHA/ACC Guideline for the management of patients with valvular heart disease. J Am Coll Cardiol, 2014; 63: e67-e185.

4. Vahanian A, Alfieri O, Andreotti O et al. Guidelines on the management of valvular heart disease (version 2012). Eur Heart J, 2012; 33: 2451-2496.

5. Hita A, Baratta S, Vaccarino G et al. Severe aortic stenosis with preserved ejection fraction and evidence of impairment in structure, myocardial strain and ventricular function: A new contribution to clinical decision making. Cardiol J, 2015; 22: 613-621.

6. Lancellotti P, Magne J, Donal E et al. Clinical outcome in asymptomatic severe aortic stenosis insights from the new proposed aortic stenosis grading classification. J Am Coll Cardiol, 2012; 59: 235-243.

7. Delgado V, Tops L, VanBommel R et al. Strain analysis in patients with severe aortic stenosis and preserved left ventricular ejection fraction undergoing surgical valve replacement. Eur Heart J, 2009; 30: 3037-3047. 